\title{
The First Multilingual Surface Realisation Shared Task (SR'18): Overview and Evaluation Results
}

\author{
Simon Mille \\ UPF, Barcelona \\ simon.mille@upf.edu
}

Yvette Graham

ADAPT Research Centre, DCU

graham.yvette@gmail.com

\author{
Anja Belz \\ University of Brighton \\ a.s.belz@brighton.ac.uk
}

\author{
Emily Pitler \\ Google Inc. \\ epitler@google.com
}

\author{
Bernd Bohnet \\ Google Inc. \\ bohnetbd@google.com
}

\author{
Leo Wanner \\ ICREA and UPF, Barcelona \\ leo.wanner@upf .edu
}

\begin{abstract}
We report results from the SR'18 Shared Task, a new multilingual surface realisation task organised as part of the ACL'18 Workshop on Multilingual Surface Realisation. As in its English-only predecessor task SR'11, the shared task comprised two tracks with different levels of complexity: (a) a shallow track where the inputs were full UD structures with word order information removed and tokens lemmatised; and (b) a deep track where additionally, functional words and morphological information were removed. The shallow track was offered in ten, and the deep track in three languages. Systems were evaluated (a) automatically, using a range of intrinsic metrics, and (b) by human judges in terms of readability and meaning similarity. This report presents the evaluation results, along with descriptions of the SR'18 tracks, data and evaluation methods. For full descriptions of the participating systems, please see the separate system reports elsewhere in this volume.
\end{abstract}

\section{Introduction and Task Overview}

Natural Language Generation (NLG) is attracting growing interest both in the form of end-toend tasks (e.g. data-to-text and text-to-text generation), and as embedded component tasks (e.g. in abstractive summarisation, dialogue-based interaction and question answering).

NLG research has been given a boost by two recent developments: the rapid spread of neural language generation techniques, and the growing availability of multilingual treebanks annotated with Universal Dependencies ${ }^{1}$ (UD), to the point

\footnotetext{
1http://universaldependencies.org/
}

where as many as 70 treebanks covering about 50 languages can now be downloaded freely. ${ }^{2}$ UD treebanks facilitate the development of applications that work potentially across all languages for which UD treebanks are available in a uniform fashion, which is a big advantage for system developers. As has already been seen in parsing, UD treebanks are also a good basis for multilingual shared tasks: a method that works for some languages may also work for others.

The SR' 18 task is to generate sentences from structures at the level of abstraction of outputs in state-of-the-art parsing, encouraging participants to explore the extent to which neural network parsing algorithms can be reversed for generation. SR'18 also addresses questions about just how suitable and useful the notion of universal dependencies-which is in the process of becoming the dominant linguistic formalism across a wide range of NLP applications, parsing in particular-is for NLG. SR'18 follows the SR'11 pilot surface realisation task for English (Belz et al., 2011) which was part of Generation Challenges 2011 (GenChal'11), the fifth round of shared-task evaluation competitions (STECs) involving the language generation tasks.

Outside of the SR tasks, just three 'deep' NLG shared tasks focusing on language generation from abstract semantic representations have been organised to date: $\mathrm{WebNLG}^{3}$ (Gardent et al., 2017), SemEval Task $9^{4}$ (May and Priyadarshi, 2017), and E2E $^{5}$ (Novikova et al., 2017). What is more, these

\footnotetext{
${ }^{2}$ See the recent parsing shared task based on UDs (Nivre and de Marneffe et al., 2016): http:// universaldependencies.org/conll17/.

${ }^{3}$ http://talcl.loria.fr/webnlg/stories/ challenge.html

${ }^{4}$ http://alt.qcri.org/semeval2017/ task $9 /$

${ }^{5}$ http: //www.macs.hw.ac.uk/ InteractionLab/E2E/
} 
tasks have only been offered for English.

As in SR'11, the Multilingual Surface Realisation shared task (SR'18) comprises two tracks with different levels of difficulty:

Shallow Track: This track starts from genuine UD structures in which word order information has been removed and tokens have been lemmatised. In other words, it starts from unordered dependency trees with lemmatised nodes that hold PoS tags and morphological information as found in the original treebank annotations. The task amounts to determining the word order and inflecting words.

Deep Track: This track starts from UD structures from which functional words (in particular, auxiliaries, functional prepositions and conjunctions) and surface-oriented morphological and syntactic information have been removed. In addition to what is required for the Shallow Track, the task in the Deep Track thus also requires reintroduction of the removed functional words and morphological features.

In the remainder of this paper, we describe the data we used in the two tracks (Section 2), and the evaluation methods we used to evaluate submitted systems (Sections 3.1 and 3.2). We then briefly introduce the participating systems (Section 4), report and discuss evaluation results (Section 5), and conclude with some discussion and a look to the future (Section 6).

\section{Data}

To create the SR'18 training and testing data, we used as data sources ten UD treebanks for which annotations of reasonable quality were available, providing $\mathrm{PoS}$ tags and morphologically relevant markup (number, tense, verbal finiteness, etc.): UD_Arabic, UD_Czech, UD_Dutch, UD_English, UD_Finnish, UD_French, UD_Italian, UD_Portuguese, UD_Russian-SynTagRus and UD_SpanishAnCora. ${ }^{6}$ We created training and test data for all ten languages for the Shallow Track, and for three of the languages, namely English, French and Spanish, for the Deep Track.

Inputs in both Shallow and Deep Tracks are trees, and are released in CoNLL-U format, with no meta-information. ${ }^{7}$ Figures 1,2 and 3 show

\footnotetext{
${ }^{6}$ universaldependencies.org

${ }^{7}$ http: //universaldependencies.org/
}

a sample original UD annotation for English, and the corresponding shallow and deep input structures derived from it.

To create inputs to the Shallow Track, the UD structures were processed as follows:

1. Word order information was removed by randomised scrambling;

2. Words were replaced by their lemmas.

For the Deep Track, the following steps were additionally carried out:

3. Edge labels were generalised into predicate/argument labels, in the PropBank/NomBank (Palmer et al., 2005; Meyers et al., 2004) fashion. That is, the syntactic relations were mapped to core (A1, A2, etc.) and non-core (AM) labels, applying the following rules: (i) the first argument is always labeled A1 (i.e. there is no external argument AO); (ii) in order to maintain the tree structure and account for some cases of shared arguments, there can be inverted argument relations; (iii) all modifier edges are assigned the same generic label $A M$; (iv) there is a coordinating relation; see the inventory of relations in Table 1 .

4. Functional prepositions and conjunctions in argument position (i.e. prepositions and conjunctions that can be inferred from other lexical units or from the syntactic structure) are removed (e.g. by and of in Figure 2); prepositions and conjunctions retained in the deep representation can be found under a $A 2 I N V$ dependency; a dependency path Gov $A M \rightarrow$ Dep A2INV $\rightarrow$ Prep is equivalent to a predicate (the conjunction/preposition) with 2 arguments: Gov $\leftarrow$ A1 Prep A2 $\rightarrow$ Dep.

5. Definite and indefinite determiners, auxiliaries and modals are converted into attribute/value pairs, as are definiteness features, and the universal aspect and mood features $^{8}$, see examples in Figure 3.

6. Subject and object relative pronouns directly linked to the main relative verb are removed (and instead, the verb is linked to the antecedent of the pronoun); a dummy pronoun

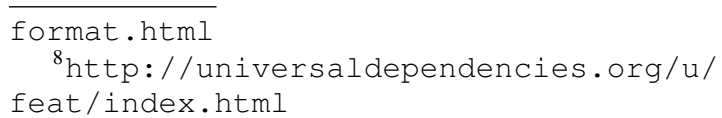



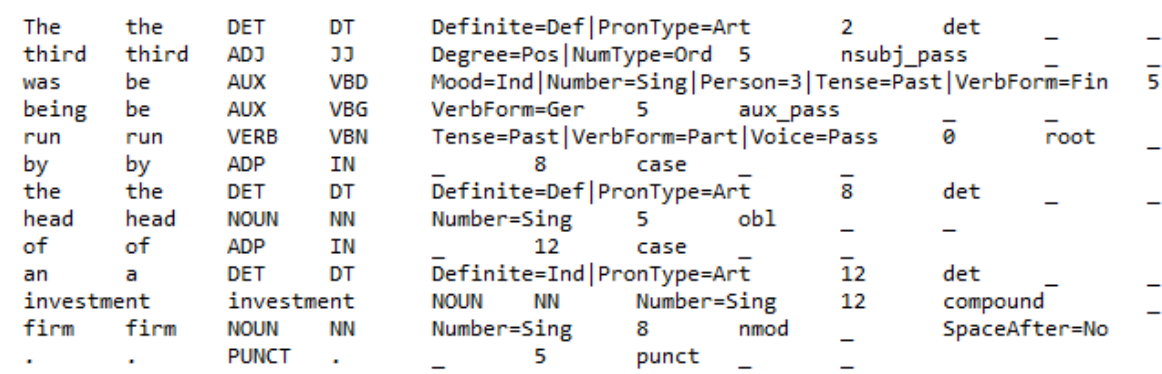

Figure 1: A sample UD structure in English.

$\begin{array}{llll}\text { the } & - & \text { DET } & \text { DT } \\ \text { third } & - & \text { ADJ } & \text { JJ } \\ \text { run } & - & \text { VERB } & \text { VBN } \\ \text { be } & - & \text { AUX } & \text { VBD } \\ \text { be } & - & \text { AUX } & \text { VBG } \\ \text { head } & - & \text { NOUN } & \text { NN } \\ \text { by } & - & \text { PUNCT } & \text { - } \\ \text { the } & - & \text { ADP } & \text { IN } \\ \text { firm } & - & \text { DET } & \text { DT } \\ \text { a } & - & \text { NOUN } & \text { NN } \\ \text { investment } & \text { DET } & \text { DT } \\ \text { of } & - & \text { ADP } & \text { NOUN }\end{array}$

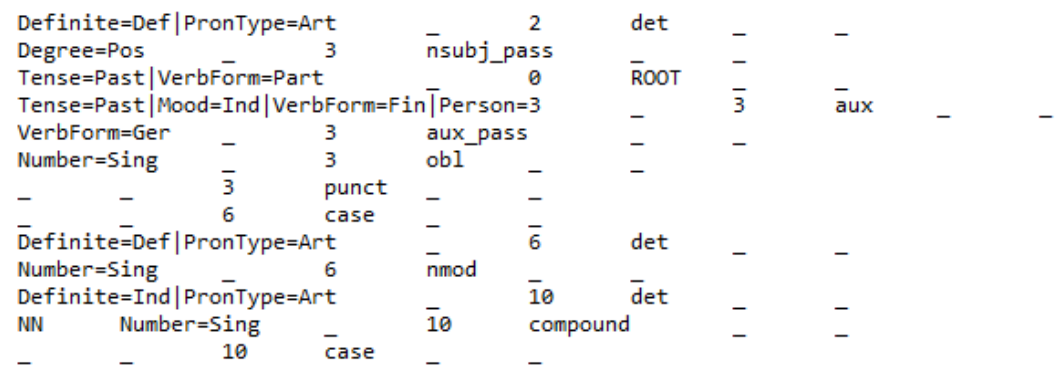

Figure 2: Shallow input (Track 1) derived from UD structure in Figure 1.

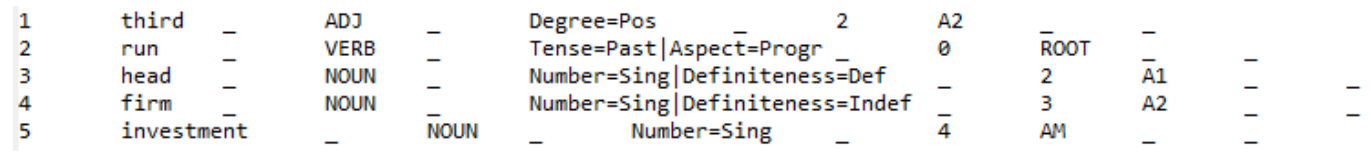

Figure 3: Deep input (Track 2) derived from UD structure in Figure 1.

node for the subject is added if an originally finite verb has no first argument and no available argument to build a passive; for a prodrop language such as Spanish, a dummy pronoun is added if the first argument is missing.

7. Surface-level morphologically relevant information as prescribed by syntactic structure or agreement (such as verbal finiteness or verbal number) is removed, whereas semantic-level information such as nominal number and verbal tense is retained.

8. Fine-grained PoS labels found in some treebanks (see e.g. column 5 in Figure 2) are removed, and only coarse-grained ones are retained (column 4 in Figures 2 and 3).

Shallow Track inputs were generated with the aid of a simple Python script from the original UD structures. During the conversion, we filtered out sentences that contained dependencies that only make sense in an analysis context (e.g. reparandum, or orphan). This amounted to around $1.5 \%$ of sentences for the different languages on average; see Table 2 for an overview of the final sizes of the datasets. Deep Track inputs were then generated by automatically processing the Shallow Track structures using a series of graphtransduction grammars that cover steps 3-8 above (in a similar fashion as Mille et al. (2017)). There is a node-to-node correspondence between the deep and shallow input structures.

The Deep Track inputs can be seen as closer to a realistic application context for NLG systems, in which the component that generates the inputs presumably would not have access to syntactic or language-specific information (see, e.g. the inputs in the SemEval, WebNLG, E2E shared tasks). At the same time, we used only information found in the UD syntactic structures to create the deep inputs, and tried to keep their structure simple. It can be argued that not all the information necessary to reconstruct the original sentences is available in the Deep Track inputs. Task definitions specifically designed for NLG, as used e.g. in SemEval Task 9, tend to use abstract meaning representations (AMRs) as inputs that contain additional information such as OntoNotes labelling or typed circumstantials, which make the generation task easier. In the SR'18 Deep Track inputs, words are not disambiguated, full prepositions may be missing, and some argument relations may be underspecified or missing. 


\begin{tabular}{|c|c|c|}
\hline Deep label & Description & Example \\
\hline $\mathrm{A} 1, \mathrm{~A} 2, \ldots, \mathrm{A} 6$ & nth argument of a predicate & fall $\rightarrow$ the ball \\
\hline $\mathrm{A} 1 \mathrm{INV}, \ldots, \mathrm{A} 6 \mathrm{INV}$ & nth inverted argument of a predicate & the ball $\rightarrow$ fall \\
\hline AM/AMINV & $\begin{array}{c}\text { (i) none of governor or dependent are argument of the other } \\
\text { (ii) unknown argument slot }\end{array}$ & fall $\rightarrow$ last night \\
\hline LIST & List of elements & fall $\rightarrow$ [and] bounce \\
\hline NAME & Part of a name & Tower $\rightarrow$ Eiffel \\
\hline DEP & Undefined dependent & N/A \\
\hline
\end{tabular}

Table 1: Deep labels.

\begin{tabular}{|c|c|c|c|c|c|c|c|c|c|c|}
\hline & ar & cs & en & es & fi & fr & it & nl & pt & ru \\
\hline \hline train & 6,016 & 66,485 & 12,375 & 14,289 & 12,030 & 14,529 & 12,796 & 12,318 & 8,325 & 48,119 \\
dev & 897 & 9,016 & 1,978 & 1,651 & 1,336 & 1,473 & 562 & 720 & 559 & 6,441 \\
test & 676 & 9,876 & 2,061 & 1,719 & 1,525 & 416 & 480 & 685 & 476 & 6,366 \\
\hline
\end{tabular}

Table 2: SR'18 dataset sizes for training, development and test sets.

\section{Evaluation Methods}

\subsection{Automatic methods}

We used BLEU, NIST, and inverse normalised character-based string-edit distance (referred to as DIST, for short, below) to assess submitted systems. BLEU (Papineni et al., 2002) is a precision metric that computes the geometric mean of the $n$-gram precisions between generated text and reference texts and adds a brevity penalty for shorter sentences. We use the smoothed version and report results for $n=4$.

NIST $^{9}$ is a related n-gram similarity metric weighted in favour of less frequent $\mathrm{n}$-grams which are taken to be more informative.

Inverse, normalised, character-based string-edit distance (DIST in the tables below) starts by computing the minimum number of character inserts, deletes and substitutions (all at cost 1) required to turn the system output into the (single) reference text. The resulting number is then divided by the number of characters in the reference text, and finally subtracted from 1 , in order to align with the other metrics. Spaces and punctuation marks count as characters; output texts were otherwise normalised as for all metrics (see below).

The figures in the tables below are the systemlevel scores for BLEU and NIST, and the mean sentence-level scores for DIST.

Text normalisation: Output texts were normalised prior to computing metrics by lowercasing all tokens, removing any extraneous whitespace characters.

\footnotetext{
${ }^{9}$ http://www.itl.nist.gov/iad/mig/tests/mt/doc/ngramstudy.pdf; http://www.itl.nist.gov/iad/mig/tests/mt/2009/
}

Missing outputs: Missing outputs were scored 0 . Since coverage was $100 \%$ for all systems except one, we only report results for all sentences (incorporating the missing-output penalty), rather than also separately reporting scores for just the in-coverage items.

\subsection{Human-assessed methods}

We assessed two quality criteria in the human evaluations, in separate evaluation experiments: Readability and Meaning Similarity. As in SR'11 (Belz et al., 2011), we used continuous sliders as rating tools, because raters tend to prefer them (Belz and Kow, 2011). Slider positions were mapped to values from 0 to 100 (best). Raters were first given brief instructions, including instructions to ignore formatting errors, superfluous whitespace, capitalisation issues, and poor hyphenation. The part of the instructions used only in the Readability assessment experiments was:

"The quality criterion you need to assess is Readability. This is sometimes called fluency, and your task is to decide how well the given text reads; is it good fluent English, or does it have grammatical errors, awkward constructions, etc.

Please rate the text by moving the slider to the position that corresponds to your rating, where 0 is the worst, and 100 is the best rating."

The corresponding instructions for Meaning Similarity assessment, in which system outputs were compared to reference sentences, were as follows:

"The quality criterion you need to assess is Meaning Similarity. You need to read 
both texts, and then decide how close in meaning the second text (in black) is to the first (in grey).

Please use the slider at the bottom of the page to express your rating. The closer in meaning the second text clipping is to the first, the further to the right (towards 100) you need to place the slider.

In other words, a rating of $100 \%$ would mean that the meaning of the two text clippings is exactly identical."

Slider design: In SR'11, a slider design was used, which had a smiley face at the 100 end and a frowning face at the 0 end, with the pointer starting out at 50. For conformity with what has emerged as a new affordable human evaluation standard over the past two years in the main machine translation shared tasks held at WMT (Bojar et al., 2017a), we changed this design to look as follows, with the pointer starting at 0 :

\section{$0 \%$}

Test data sets for human evaluations: Test set sizes out of the box varied considerably for the different languages. For the human test sets we selected either the entire set or a subset of 1,000, whichever was the smaller number, for a given language. For subsets, test set items were selected randomly but ensuring a similar sentence length distribution as in the whole set.

Reported scores: Again in keeping with the WMT approach, we report both average raw scores and average standardised scores per system. In order to produce standardised scores we simply map each individual evaluator's scores to their standard scores (or z-scores) computed on the set of all raw scores by the given evaluator using each evaluator's mean and standard deviation. For both raw and standard scores, we compute the mean of sentence-level scores.

\subsubsection{Mechanical Turk evaluations}

For three of the languages in the shallow track (English, Spanish and French), we replicated the human evaluation method from WMT'17, known as Direct Assessment (DA) (Graham et al., 2016), exactly, except that we also ran (separate) experiments to assess the Readability criterion, using the same method.
Quality assurance: System outputs are randomly assigned to HITs (following Mechanical Turk terminology) of 100 outputs, of which 20 are used solely for quality assurance (QA) (i.e. do not count towards system scores): (i) some are repeated as are, (ii) some are repeated in a 'damaged' version and (iii) some are replaced by their corresponding reference texts. In each case, a minimum threshold has to be reached for the HIT to be accepted: for (i), scores must be similar enough, for (ii) the score for the damaged version must be worse, and for (iii) the score for the reference text must be high. For full details of how these additional texts are created and thresholds applied, please refer to Bojar et al. (2017a). Below we report QA figures for the MTurk evaluations (Section 3.2.1).

Code: We were able to reuse, with minor adaptations, the code produced for the WMT'17 evaluations. $^{10}$

\subsubsection{Google Data Compute Evaluation}

In order to cover more languages, and to enable comparison between crowdsourced and expert evaluation, we also conducted human evaluations using Google's internal 'Data Compute' system evaluation service, where experienced evaluators carefully assess each system output. We used an interface that matches the WMT'17 interface above, as closely as was possible within the constraints of the Data Compute platform.

Everything stated at the beginning of Section 3.2 also holds for the expert annotator evaluations with Google Data Compute.

Quality assurance: Because in the Google Data Compute version of the evaluation experiment we were using expert evaluators from a pool of workers routinely employed to perform such tasks, we did not replicate the WMT'17 QA techniques precisely, opting for a simpler test of self-consistency, or intra-evaluator agreement (IEA) instead. Test set items were randomly grouped into sets of 100 (which we are also calling HITs here for uniformity) and order was again randomised before presentation to evaluators. Each evaluator did at least one HIT. Each HIT contained 5 items which were duplicated to test for IEA which we computed as the average Pearson correlation coefficient per HIT. The average IEA for English was 0.75 on the

\footnotetext{
${ }^{10} \mathrm{https} / /$ github.com/ygraham/segment-mteval
} 
raw scores for Meaning Similarity, and 0.66 for Readability.

\section{Overview of Submitted Systems}

Eight different teams (out of twenty-one registered) submitted outputs to SR'18: the ADAPT Centre (ADAPT, Ireland), AX Semantics (AX, Germany), IIT-BHU Varanasi (IIT-BHU, India), Ohio State University (OSU, USA), University of São Paulo (NILC, Brazil), Tilburg University (Tilburg, The Netherlands), Università degli Studi di Torino (DipInfo-UniTo, Italy), and Technische Universität Darmstadt (BinLin, Germany).

All teams submitted outputs for at least the English Shallow Track; one team participated in the Deep Track (ADAPT, English), and three teams submitted outputs for all ten languages of the Shallow Track (AX, OSU, and BinLin). Most submitted systems are based on neural components, and break down the surface realisation task into two subtasks: linearisation, and word inflection. Details of each approach are provided in the teams' reports elsewhere in this volume; here, we briefly summarise each approach:

ADAPT uses linearised parse tree inputs to train a sequence-to-sequence LSTM model with copy attention, augmenting the training set with additional synthetic data.

$\mathbf{A X}$ is trained on word pairs for ordering and is combined with a rule-based morphology component.

IIT-BHU uses an LSTM-based encoderdecoder model for word re-inflection, and a Language Model-based approach for word reordering.

OSU first generates inflected wordforms with a neural sequence-to-sequence model, and then incrementally linearises them using a global linear model over features that take into account the dependency structure and dependency location. ${ }^{11}$

NILC is a neural-based system that uses a bottom-up approach to build the sentence using the dependency relations together with a language model, and language-specific lexicons to produce the word forms of each lemma in the sentence.

Tilburg works by first preprocessing an input dependency tree into an ordered linearised string,

\footnotetext{
${ }^{11}$ Some of OSU's outputs were submitted after the start of the human evaluations and are not included in this report; outputs submitted late, but before the human evaluation started, are included and marked with asterisks in the results tables.
}

which is then realised using a statistical machine translation model.

DipInfo-UniTo employs two separate neural networks with different architectures to predict the word ordering and the morphological inflection independently; outputs are combined to produce the final sentence.

BinLin uses one neural module as a binary classifier in a sequential process of ordering token lemmas, and another for character-level morphology generation where the words are inflected to finish the surface realisation.

\section{Evaluation results}

\subsection{Results from metric evaluations}

Tables 3-5 show BLEU-4, NIST, and DIST results for both the Shallow and Deep tracks, for all submitted systems; results are listed in order of number of languages submitted for. Best results for each language are shown in boldface.

In terms of BLEU-4, in the Shallow Track, Tilburg obtained the best scores for four languages (French, Italian, Dutch, Portuguese), OSU for three (Arabic, Spanish, Finnish), BinLin for two (Czech, Russian), and ADAPT for one (English). The highest BLEU-4 scores across languages were obtained on the English and Spanish datasets, with BLEU-4 scores of 69.14 (ADAPT) and 65.31 (OSU) respectively.

Results are identical for DIST, except that AX, rather than BinLin, has the highest score for Czech. The picture for NIST is also very similar to that for BLEU-4, except that ADAPT and OSU are tied for best NIST score for English, and BinLin (rather than Tilburg) has the best NIST score for Dutch.

In the Deep Track, only ADAPT submitted system outputs (English), and as expected, the scores are much lower than for the Shallow Track, across all metrics.

\subsection{Results from human evaluations}

Given the small number of submissions in the Deep Track, we conducted human evaluations for the Shallow Track only. We used Mechanical Turk for the three languages for which this is feasible (English, Spanish and French), and our aim was to also conduct evaluations via Google's Data Compute service for three additional languages which had the next highest numbers of submissions, as 


\begin{tabular}{|c|c|c|c|c|c|c|c|c|c|c||c|}
\hline & \multicolumn{9}{|c|}{ Shallow } & Deep \\
\cline { 2 - 13 } & ar & cs & en & es & fi & fr & it & nl & pt & ru & en \\
\hline \hline AX & 4.57 & 9.75 & 28.09 & 10.2 & 7.95 & 7.87 & 16.35 & 14.21 & 16.29 & 15.59 & - \\
\hline BinLin & 16.2 & $\mathbf{2 5 . 0 5}$ & 29.6 & 32.15 & 23.26 & 20.53 & 23.55 & 22.69 & 24.59 & $\mathbf{3 4 . 3 4}$ & - \\
\hline OSU & $\mathbf{2 5 . 6 5}$ & - & 66.33 & $\mathbf{6 5 . 3 1}$ & $\mathbf{3 7 . 5 2} *$ & $38.24^{*}$ & - & $25.52^{*}$ & - & - & - \\
\hline Tilburg & - & - & 55.29 & 49.47 & - & $\mathbf{5 2 . 0 3}$ & $\mathbf{4 4 . 4 6}$ & $\mathbf{3 2 . 2 8}$ & $\mathbf{3 0 . 8 2}$ & - & - \\
\hline DipInfo & - & - & 23.2 & 26.9 & - & 23.12 & 24.61 & - & - & - & - \\
\hline NILC & - & - & 50.74 & 51.58 & - & - & - & - & 27.12 & - & - \\
\hline ADAPT & - & - & $\mathbf{6 9 . 1 4}$ & - & - & - & - & - & - & - & $\mathbf{2 1 . 6 7}$ \\
\hline IIT-BHU & - & - & 8.04 & - & - & - & - & - & - & - & - \\
\hline
\end{tabular}

Table 3: BLEU-4 scores for the test data. Bold = best score per language. $*=$ late submission.

\begin{tabular}{|c|c|c|c|c|c|c|c|c|c|c|c||c|}
\hline & \multicolumn{9}{|c|}{ Shallow } & Deep \\
\cline { 2 - 14 } & ar & cs & en & es & fi & fr & it & nl & pt & ru & en \\
\hline \hline AX & 5.13 & 9.33 & 9.51 & 8.26 & 6.84 & 6.45 & 6.83 & 7.81 & 6.78 & 9.93 & - \\
\hline BinLin & 6.94 & $\mathbf{1 0 . 7 4}$ & 9.58 & 10.21 & 9.36 & 7.21 & 7.6 & $\mathbf{8 . 6 4}$ & 7.54 & $\mathbf{1 3 . 0 6}$ & - \\
\hline OSU & $\mathbf{7 . 1 5}^{*}$ & - & $\mathbf{1 2 . 0 2}$ & $\mathbf{1 2 . 7 4}$ & $\mathbf{9 . 5 6}^{*}$ & $8.00^{*}$ & - & $7.33^{*}$ & - & - & - \\
\hline Tilburg & - & - & 10.86 & 11.12 & - & $\mathbf{9 . 8 5}$ & $\mathbf{9 . 1 1}$ & 8.05 & $\mathbf{7 . 5 5}$ & - & - \\
\hline DipInfo & - & - & 8.86 & 9.58 & - & 7.72 & 8.25 & - & - & - & - \\
\hline NILC & - & - & 10.62 & 11.17 & - & - & - & - & 7.56 & - & - \\
\hline ADAPT & - & - & $\mathbf{1 2 . 0 2}$ & - & - & - & - & - & - & - & $\mathbf{6 . 9 5}$ \\
\hline IIT-BHU & - & - & 7.71 & - & - & - & - & - & - & - & - \\
\hline
\end{tabular}

Table 4: NIST scores for the test data. Bold $=$ best score per language. $*=$ late submission.

\begin{tabular}{|c|c|c|c|c|c|c|c|c|c|c||c|}
\hline & \multicolumn{9}{|c|}{ Shallow } & Deep \\
\cline { 2 - 14 } & ar & cs & en & es & fi & fr & it & nl & pt & ru & en \\
\hline \hline AX & 38.96 & $\mathbf{3 6 . 4 8}$ & 70.01 & 21.12 & 35.59 & 22.3 & 40.96 & 49.65 & 51.7 & 34.28 & - \\
\hline BinLin & 44.37 & 35.7 & 65.9 & 36.95 & 41.21 & 28.6 & 40.74 & 48.23 & 51.36 & $\mathbf{3 4 . 5 6}$ & - \\
\hline OSU & $\mathbf{4 6 . 4 9} *$ & - & 70.22 & $\mathbf{6 1 . 4 6}$ & $\mathbf{5 8 . 7} *$ & $53.69^{*}$ & - & $57.77 *$ & - & - & - \\
\hline Tilburg & - & - & 79.29 & 51.73 & - & $\mathbf{5 5 . 5 4}$ & $\mathbf{5 8 . 6 1}$ & $\mathbf{5 7 . 8 1}$ & $\mathbf{6 0 . 7}$ & - & - \\
\hline DipInfo & - & - & 51.87 & 24.53 & - & 18.04 & 36.11 & - & - & - & - \\
\hline NILC & - & - & 77.56 & 53.78 & - & - & - & - & 57.43 & - & - \\
\hline ADAPT & - & - & $\mathbf{8 0 . 4 2}$ & - & - & - & - & - & - & - & $\mathbf{4 8 . 6 9}$ \\
\hline IIT-BHU & - & - & 47.63 & - & - & - & - & - & - & - & - \\
\hline
\end{tabular}

Table 5: DIST scores for the test data. Bold $=$ best score per language. $*=$ late submission.

well as for English in order to enable us to compare results obtained with the two different methods. However, most of the latter evaluations are still ongoing and will be reported separately in a future paper. Below, we report Google Data Compute results and comparisons with Mechanical Turk results, for English only.

\subsubsection{Mechanical Turk results}

Tables 6, 7 and 8 show the results of the human evaluation carried out via Mechanical Turk with Direct Assessment (MTurk DA), for English, French and Spanish, respectively. See Section 3.2 for details of the evaluation method. 'DA' refers to the specific way in which scores are collected in the WMT approach which differs from what we did for SR'11, and here in the Google Data Compute experiments.

English: Average Meaning Similarity DA scores for English systems range from $86.9 \%$ to
$67 \%$ with OSU achieving the highest overall score in terms of both average raw DA scores and corresponding z-scores. Readability scores for the same set of systems range from $78.7 \%$ to $41.3 \%$, revealing that MTurk workers rate the Meaning Similarity between generated texts and corresponding reference sentences higher in general than Readability. In order to investigate how Readability of system outputs compare to human-produced text, we included the original test sentences as a system in the Readability evaluation (for Meaning Similarity the notional score is 100\%). Unsurprisingly, human text achieves the highest score in terms of Readability (78.7\%) but is quite closely followed by the best performing system in terms of Readability, ADAPT (73.9\%).

Overall in the English Shallow Track, average DA scores for systems are close. We tested for statistical significance of differences between average DA scores using a Wilcoxon rank sum test. 
Figure 4 shows significance test results for each pair of systems participating in the English evaluation in the form of heatmaps where a green cell denotes a significantly higher average score for the system in that row over the system in that column, with a darker shade of green denoting a conclusion drawn with more certainty. Results show that two entries are tied for first place in terms of Meaning Similarity, OSU and ADAPT, with the small difference in average scores proving not statistically significant. In terms of Readability, however, the ADAPT sentences achieve a significantly higher readability score compared to OSU.

French: Table 7 shows average DA scores for systems participating in the French Shallow Track. Meaning Similarity scores for French systems range from $72.9 \%$ to $48.6 \%$ with the Tilburg system achieving the highest overall score. In terms of Readability, again Tilburg achieves the highest average score of $65.4 \%$, with a considerable gap to the next best entry, OSU. Compared to the human results, there is a larger gap than we saw for English outputs.

Figure 5 shows results of tests for statistical significance between average DA scores for systems in the French Shallow Track. Tilburg achieves a significantly higher average DA score compared to all other systems in terms of both Meaning Similarity and Readability. All systems are significantly worse in terms of Readability than the human authored texts.

Spanish: Table 8 shows average DA scores for systems participating in the Shallow Track for Spanish. Meaning Similarity scores range from $77.3 \%$ to $43.9 \%$, with OSU achieving the highest score. In terms of Readability, the text produced by the systems ranges from $77.0 \%$ to $33.0 \%$, and again OSU achieves the highest score. Figure 6 shows results of the corresponding significance tests: OSU significantly outperforms all other participating systems with respect to both evaluation criteria. Human-generated texts are significantly more readable than all system outputs.

MTurk DA quality control: Only $31 \%$ of workers passed quality control (being able to replicate scores for same sentences and scoring damaged sentences lower, for full details see Bojar et al., 2017a), highlighting the danger of crowdsourcing without good quality control measures. The remaining $69 \%$, who did not meet this criterion, were omitted from computation of the of- ficial DA results above. Of those $31 \%$ included in the evaluation, a very high proportion, $97 \%$, showed no significant difference in scores collected in repeated assessment of the same sentences; these high levels of agreement are consistent with what we have seen in DA used for Machine Translation (Graham et al., 2016) and Video Captioning evaluation (Graham et al., 2017).

Agreement with automatic metrics: Table 9 shows Pearson correlations between MTurk DA scores and automatic metric scores in the English, French and Spanish shallow tracks. Overall, BLEU agrees most consistently across the different tasks, achieving a correlation above 0.95 in all settings, whereas the correlation of NIST scores with human Meaning Similarity scores is just 0.854 for French, while DIST scores correlate with human Readability scores at just 0.831 for English.

Conclusions from metric correlations should be drawn with a degree of caution, since in all cases the sample size from which we compute correlations is small, 8 systems for English, 5 for French, and 6 for Spanish. We carried out significance tests to investigate to what degree differences in correlations are likely to occur by chance. In order to take into account the fact that we are comparing correlations between human assessment and competing pairs of metrics (where metric scores themselves correlate with each other), we apply a Williams test for significance of differences in dependent correlations, as done in evaluation of Machine Translation metrics (Graham and Baldwin, 2014; Bojar et al., 2017b).

Results are shown in Table 9. Correlations between metrics and human assessment in bold are not significantly lower than any other metric. As can be seen from Table 9, there is no significant difference between any of the three metrics in terms of correlation with human assessment in both the French and Spanish tracks. In the English track, however, the correlation of BLEU and NIST scores with human assessment are significantly higher than that of DIST.

\subsubsection{Google Data Compute results}

Table 10 shows the results for the English assessment conducted via the Google Data Compute (GDC) evaluation service with expert evaluators.

One difference between the MTurk and the Google results is the range of scores, which for 


\begin{tabular}{rrrrl}
\hline \multicolumn{5}{c}{ Meaning Similarity } \\
$\%$ & $z$ & $n$ & Assess. & System \\
\hline \multicolumn{6}{c}{} & & & \\
86.9 & 0.369 & 1,249 & 1,422 & OSU \\
85.5 & 0.314 & 1,238 & 1,429 & ADAPT \\
84.8 & 0.291 & 1,294 & 1,498 & Tilburg \\
84.2 & 0.280 & 1,229 & 1,407 & NILC \\
77.5 & 0.043 & 1,256 & 1,442 & AX \\
75.8 & 0 & 1,264 & 1,462 & BinLin \\
72.6 & -0.120 & 1,244 & 1,427 & DipInfo \\
67.0 & -0.312 & 1,257 & 1,412 & IIT-BHU \\
\hline
\end{tabular}

\begin{tabular}{rrrrl}
\hline \multicolumn{5}{c}{ Readability } \\
$\%$ & $z$ & $n$ & Assess. & System \\
\hline 78.7 & 0.797 & 831 & 1,350 & HUMAN \\
73.9 & 0.638 & 1,065 & 1,301 & ADAPT \\
71.2 & 0.558 & 1,117 & 1,374 & OSU \\
62.1 & 0.258 & 1,109 & 1,377 & Tilburg \\
58.1 & 0.166 & 1,086 & 1,342 & NILC \\
52.5 & -0.019 & 1,080 & 1,343 & AX \\
50.1 & -0.102 & 1,076 & 1,336 & BinLin \\
42.7 & -0.345 & 1,091 & 1,355 & DipInfo \\
41.3 & -0.376 & 1,081 & 1,296 & IIT-BHU \\
\hline
\end{tabular}

Table 6: MTurk DA human evaluation results for English Shallow Track; \% = average DA score (0-100); $z=\mathrm{z}$-score; $n=$ number of distinct sentences assessed; Assess. $=$ total number of sentences assessed.

\begin{tabular}{rrrrl}
\hline \multicolumn{5}{c}{ Meaning Similarity } \\
$\%$ & $z$ & $n$ & Assess. & System \\
\hline & & & & \\
72.9 & 0.365 & 416 & 1,651 & Tilburg \\
69.1 & 0.237 & 416 & 1,570 & OSU \\
58.9 & -0.133 & 416 & 1,575 & BinLin \\
52.8 & -0.32 & 416 & 1,648 & DipInfo \\
48.6 & -0.444 & 416 & 1,592 & AX \\
\hline
\end{tabular}

\begin{tabular}{rrrrl}
\hline \multicolumn{4}{c}{ Readability } \\
$\%$ & $z$ & $n$ & Assess. & System \\
\hline 89.9 & 1.525 & 218 & 650 & HUMAN \\
65.4 & 0.607 & 416 & 1060 & Tilburg \\
54.7 & 0.179 & 416 & 1007 & OSU \\
41.5 & -0.26 & 416 & 1031 & BinLin \\
38.7 & -0.456 & 416 & 1094 & DipInfo \\
32.9 & -0.659 & 416 & 1033 & AX \\
\hline
\end{tabular}

Table 7: MTurk DA human evaluation results for French Shallow Track; $\%=$ average DA score (0-100); $z=\mathrm{z}$-score; $n=$ number of distinct sentences assessed; Assess. $=$ total number of sentences assessed.

\begin{tabular}{rrrrl}
\hline \multicolumn{5}{c}{ Meaning Similarity } \\
$\%$ & $z$ & $n$ & Assess. & System \\
\hline & & & & \\
77.3 & 0.519 & 1,255 & 1,502 & OSU \\
66.8 & 0.175 & 1,231 & 1,439 & NILC \\
65.7 & 0.136 & 1,190 & 1,401 & Tilburg \\
54.9 & -0.214 & 1,202 & 1,395 & BinLin \\
48.4 & -0.445 & 1,190 & 1,401 & DipInfo \\
43.9 & -0.583 & 1,225 & 1,449 & AX \\
\hline
\end{tabular}

\begin{tabular}{rrrrl}
\hline \multicolumn{5}{c}{ Readability } \\
DA & $z$ & $n$ & Assess. & System \\
\hline 89.6 & 1.120 & 889 & 1,237 & HUMAN \\
77.0 & 0.731 & 1,399 & 1,691 & OSU \\
63.1 & 0.265 & 1,371 & 1,645 & Tilburg \\
57.2 & 0.093 & 1,384 & 1,631 & NILC \\
45.1 & -0.299 & 1,367 & 1,625 & BinLin \\
36.9 & -0.558 & 1,370 & 1,629 & DipInfo \\
33.0 & -0.700 & 1,371 & 1,657 & AX \\
\hline
\end{tabular}

Table 8: MTurk DA human evaluation results for Spanish Shallow Track; $\%=$ average DA score $(0-100)$; $z=\mathrm{z}$-score; $n=$ number of distinct sentences assessed; Assess. $=$ total number of sentences assessed.

\begin{tabular}{llcccc}
\hline & & Meaning Sim. & BLEU & NIST & DIST \\
\hline \multirow{2}{*}{ English } & Meaning Sim. & & $\mathbf{0 . 9 6 8}$ & $\mathbf{0 . 9 6 7}$ & 0.911 \\
& Readability & 0.927 & $\mathbf{0 . 9 7 1}$ & $\mathbf{0 . 9 7 7}$ & 0.831 \\
\hline \multirow{2}{*}{ French } & Meaning Sim. & & $\mathbf{0 . 9 5 4}$ & $\mathbf{0 . 8 5 4}$ & $\mathbf{0 . 9 6 8}$ \\
& Readability & 0.984 & $\mathbf{0 . 9 7 8}$ & $\mathbf{0 . 9 2 4}$ & $\mathbf{0 . 9 3 8}$ \\
\hline \multirow{2}{*}{ Spanish } & Meaning Sim. & & $\mathbf{0 . 9 8 6}$ & $\mathbf{0 . 9 8 0}$ & $\mathbf{0 . 9 9 0}$ \\
& Readability & 0.989 & $\mathbf{0 . 9 6 9}$ & $\mathbf{0 . 9 7 1}$ & $\mathbf{0 . 9 6 9}$ \\
\hline
\end{tabular}

Table 9: Pearson correlation of DA human evaluation scores with Automatic Metrics for English, French and Spanish Shallow Track. 
Meaning Similarity

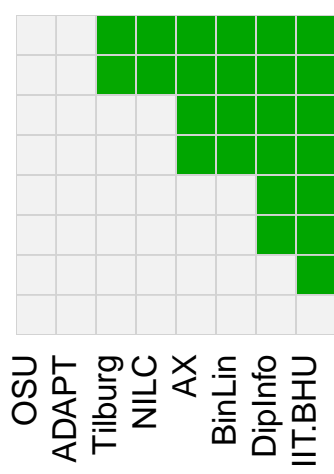

Readability

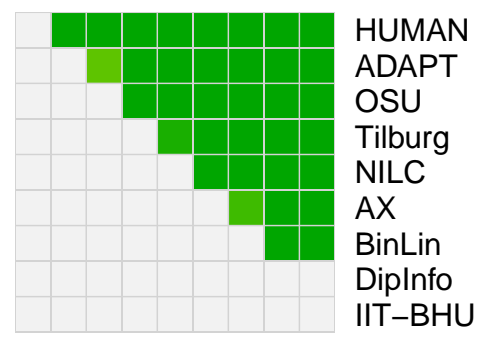

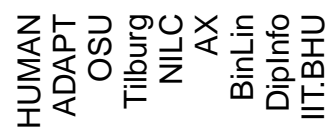

Figure 4: MTurk DA human evaluation significance test results for the English shallow track.
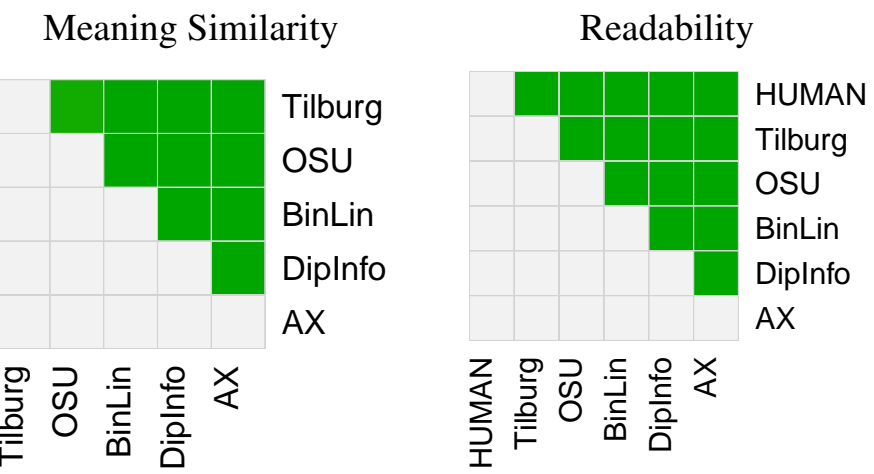

Figure 5: MTurk DA human evaluation significance test results for the French shallow track.
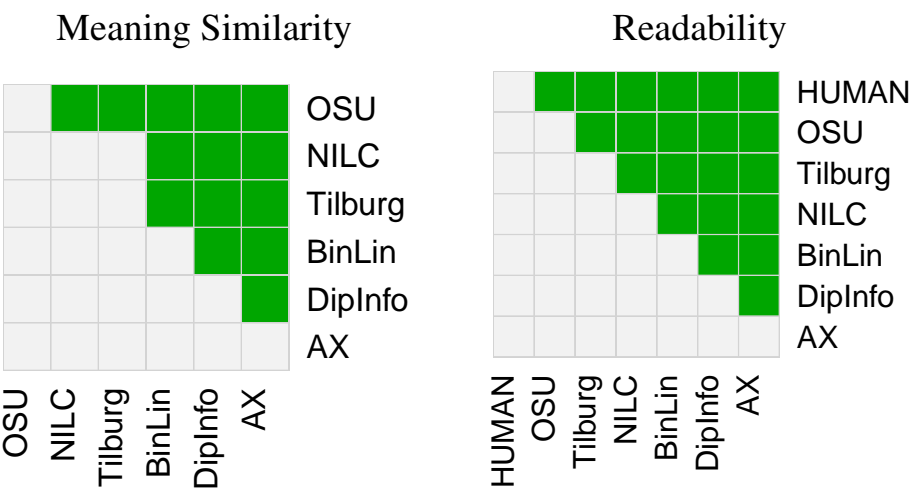

Figure 6: MTurk DA human evaluation significance test results for the Spanish shallow track.

Meaning Similarity range from 67 to 86.9 for MTurk, compared to 52 to 86.1 for GDC. The latter is a wider range of scores, and expert evaluators' scores distinguish between systems more clearly than the crowdsourced scores which place the top four systems very close together.

Readability scores range from 41.3 to 78.7 for MTurk, and from 60.2 to 88.2 for GDC. The expert evaluators tended to assign higher scores overall, but their range and the way they distinguish between systems is similar. For example, neither evaluation found much difference for the bottom two systems.

The rank order of systems in the two separate evaluations is identical. Table 11 shows the Pearson correlation of scores for systems in the evaluations, where meaning similarity scores correlate almost perfectly at 0.997 (raw \%) and $0.993(z)$ and readability at 0.986 (raw \%) and $0.985(z)$.

\section{Conclusion}

SR'18 was the second surface realisation shared task, and followed an earlier pilot task for English, SR'11. Participation was high for a first instance 


\begin{tabular}{rrrl}
\hline \multicolumn{4}{c}{ Meaning Similarity } \\
$\%$ & $z$ & $n$ & System \\
\hline 86.1 & 0.479 & 1000 & OSU \\
83.8 & 0.394 & 1000 & ADAP \\
81.8 & 0.308 & 1000 & Tilburg \\
78.8 & 0.219 & 1000 & NILC \\
68.7 & -0.109 & 1000 & AX \\
65.4 & -0.238 & 1000 & BinLin \\
59.7 & -0.414 & 1000 & DipInf \\
52.0 & -0.640 & 1000 & IIT-BH \\
\hline \multicolumn{5}{c}{ Compute human eval } \\
(0-100) for generated sentences; \\
\multicolumn{4}{c}{0.986} \\
0.997 & \multicolumn{4}{c}{0.985} \\
\hline
\end{tabular}

Table 11: Pearson correlation between human evaluations carried out using MTurk DA and Google Data Compute.

of a shared task, at least in the Shallow Track, indicating that interest is high enough to continue running it again next year to enable more teams to participate.

One important question that needs to be addressed is to what extent UDs are suitable inputs for NLG systems. More specifically, can they reasonably be expected to be generated by other, content-determining, modules in an NLG system, do they provide all the information necessary to generate surface realisations, and if not, how can they be augmented to provide it.

We hope to discuss these and related issues with the research community as we prepare the next instance of the SR Task. A goal to aim for may be to make it possible for different NLG components to be connected via standard interface representations, to increase re-usability for NLG components. However, what may constitute a good interface representation for surface realisation remains far from clear.

\section{Acknowledgments}

The work reported in this paper has been partly supported by Science Foundation Ireland (sfi.ie) under the SFI Research Centres Programme co-funded under the European Regional

\begin{tabular}{rrrl}
\hline \multicolumn{4}{c}{ Readability } \\
$\%$ & $z$ & $n$ & System \\
\hline 88.2 & 0.530 & 1000 & ADAPT \\
86.1 & 0.459 & 1000 & OSU \\
81.0 & 0.276 & 1000 & Tilburg \\
78.0 & 0.156 & 1000 & NILC \\
67.7 & -0.194 & 1000 & AX \\
65.9 & -0.299 & 1000 & BinLin \\
60.7 & -0.449 & 1000 & DipInfo \\
60.2 & -0.480 & 1000 & IIT-BHU \\
\hline
\end{tabular}

Development Fund, grant number 13/RC/2106 (ADAPT Centre for Digital Content Technology, www.adaptcentre.ie) at Dublin City University, and by the European Commission in the framework of the H2020 Programme under the contract numbers 779962-RIA, 700475-IA, 7000024-RIA, and 645012RIA.

\section{References}

Anja Belz and Eric Kow. 2011. Discrete vs. continuous rating scales for language evaluation in NLP. In Proceedings of the 49th Annual Meeting of the Association for Computational Linguistics (ACLHLT'11).

Anja Belz, Michael White, Dominic Espinosa, Eric Kow, Deirdre Hogan, and Amanda Stent. 2011. The first surface realisation shared task: Overview and evaluation results. In Proceedings of the 13th European Workshop on Natural Language Generation, ENLG '11, pages 217-226, Stroudsburg, PA, USA. Association for Computational Linguistics.

Ondřej Bojar, Rajen Chatterjee, Christian Federmann, Yvette Graham, Barry Haddow, Shujian Huang, Matthias Huck, Philipp Koehn, Qun Liu, Varvara Logacheva, Christof Monz, Matteo Negri, Matt Post, Raphael Rubino, Lucia Specia, and Marco Turchi. 2017a. Findings of the 2017 conference on machine translation (WMT'17). In Proceedings of the Second Conference on Machine Translation, Volume 2: Shared Task Papers, pages 169214, Copenhagen, Denmark. Association for Computational Linguistics.

Ondřej Bojar, Yvette Graham, and Amir Kamran. 2017b. Results of the wmt17 metrics shared task. In Proceedings of the Second Conference on Machine Translation, Volume 2: Shared Task Papers, pages 489-513, Copenhagen, Denmark. Association for Computational Linguistics. 
Claire Gardent, Anastasia Shimorina, Shashi Narayan, and Laura Perez-Beltrachini. 2017. Creating training corpora for micro-planners. In Proceedings of the 55th Annual Meeting of the Association for Computational Linguistics (Volume 1: Long Papers), Vancouver, Canada. Association for Computational Linguistics.

Yvette Graham, George Awad, and Alan Smeaton. 2017. Evaluation of Automatic Video Captioning Using Direct Assessment. ArXiv e-prints.

Yvette Graham and Timothy Baldwin. 2014. Testing for significance of increased correlation with human judgment. In Proceedings of the 2014 Conference on Empirical Methods in Natural Language Processing (EMNLP), pages 172-176, Doha, Qatar. Association for Computational Linguistics.

Yvette Graham, Timothy Baldwin, Alistair Moffat, and Justin Zobel. 2016. Can machine translation systems be evaluated by the crowd alone. Natural Language Engineering, FirstView:1-28.

Jonathan May and Jay Priyadarshi. 2017. Semeval2017 task 9: Abstract meaning representation parsing and generation. In Proceedings of the 11th International Workshop on Semantic Evaluation (SemEval-2017), pages 534-543, Vancouver, Canada. Association for Computational Linguistics.

Adam Meyers, R. Reeves, C. Macleod, R. Szekely, V. Zielinska, B. Young, and R. Grishman. 2004. The NomBank project: An interim report. In HLTNAACL 2004 Workshop: Frontiers in Corpus Annotation, Boston, MA, May 2004, pages 24-31.

Simon Mille, Roberto Carlini, Ivan Latorre, and Leo Wanner. 2017. Upf at epe 2017: Transduction-based deep analysis. In Shared Task on Extrinsic Parser Evaluation (EPE 2017), pages 80-88, Pisa, Italy.

Joakim Nivre and Marie-Catherine de Marneffe et al. 2016. Universal dependencies v1: A multilingual treebank collection. In Proceedings of LREC, Portorož, Slovenia.

Jekaterina Novikova, Ondrej Dušek, and Verena Rieser. 2017. The E2E dataset: New challenges for endto-end generation. In Proceedings of the 18th Annual Meeting of the Special Interest Group on Discourse and Dialogue, Saarbrücken, Germany. ArXiv:1706.09254.

Martha Palmer, Daniel Gildea, and Paul Kingsbury. 2005. The proposition bank: An annotated corpus of semantic roles. Computational Linguistics, 31(1):71-105.

K. Papineni, S. Roukos, T. Ward, and W. j. Zhu. 2002. BLEU: A method for automatic evaluation of machine translation. In Proc. 40th Annual Meeting on Association for Computational Linguistics, pages 311-318, Philadelphia, Pennsylvania. 